\title{
Addition of estimated cardiorespiratory fitness to the clinical assessment of 10-year coronary heart disease risk in asymptomatic men
}

\author{
Jennifer C. Gander ${ }^{\mathrm{a}, *}$, Xuemei Sui ${ }^{\mathrm{b}}$, James R. Hébert ${ }^{\mathrm{c}, \mathrm{d}}$, Carl J. Lavie ${ }^{\mathrm{e}}$, Linda J. Hazlett ${ }^{\mathrm{c}}$, Bo Cai ${ }^{\mathrm{c}}$, Steven N. Blair ${ }^{\mathrm{b}}$ \\ a Department of Surgery, Emory University School of Medicine, Atlanta, GA, United States \\ ${ }^{\mathrm{b}}$ Department of Exercise Science, Arnold School of Public Health, University of South Carolina, Columbia, SC, United States \\ c Department of Epidemiology and Biostatistics, Arnold School of Public Health, University of South Carolina, Columbia, SC, United States \\ d South Carolina Statewide Cancer Prevention and Control Program, University of South Carolina, Columbia, SC, United States \\ e Department of Cardiovascular Diseases, John Ochsner Heart and Vascular Institute, Ochsner Clinical School-the University of Queensland School of Medicine, New Orleans, LA, United States
}

\section{A R T I C L E I N F O}

\section{Article history:}

Received 1 May 2017

Accepted 15 May 2017

Available online 18 May 2017

\section{Keywords:}

Cardiorespiratory fitness

Cardiovascular disease

Chronic disease

Exercise capacity

Framingham risk score

Men

Risk

\begin{abstract}
A B S T R A C T
The Framingham Risk Score (FRS) was developed to quantify a patient's coronary heart disease (CHD) risk. Nonexercise estimated CRF (e-CRF) may provide a clinically practical method for describing cardiorespiratory fitness. We computed e-CRF and tested its association with the FRS and CHD.

Male participants $(n=29,854)$ in the Aerobics Center Longitudinal Study (ACLS) who completed a baseline examination between 1979-2002 were followed for 12 years to determine incident CHD defined by self-report of myocardial infarction, revascularization, or CHD mortality. e-CRF was defined from a 7-item scale and categorized using age-specific tertiles. Multivariable survival analysis determined associations between FRS, e-CRF, and CHD. Interaction between e-CRF and FRS was tested by stratified analysis by 'low' and 'moderate or high' 10-year CHD risk.

Men with high e-CRF were significantly ( $\mathrm{p}$-value $<0.0001$ ) younger, and less likely to be smokers, compared to men with low e-CRF. Multivariable survival analysis reported men with high e-CRF were $29 \%$ (HR $=0.71 ; 95 \%$ $0.56,0.88$ ) less likely to experience a CHD event compared to men with low e-CRF. Stratified analyses showed men with 'low' 10-year FRS predicted CHD risk and high e-CRF had a 28\% (HR $=0.72$; 95\% CI 0.57, 0.91) lower CHD-mortality risk compared to men with low e-CRF, no association was found in this group and men with moderate e-CRF. Men who were more fit had a decreased risk for CHD compared to men in the lowest third of fitness. Estimated CRF may add clinical value to the FRS and help clinicians better predict long-term CHD risk.
\end{abstract}

(c) 2017 The Authors. Published by Elsevier Inc. This is an open access article under the CC BY-NC-ND license

(http://creativecommons.org/licenses/by-nc-nd/4.0/).

\section{Introduction}

Despite the decrease in coronary heart disease (CHD) incidence worldwide in the past 30 years (Rodriguez et al., 2006; Bennett et al., 2006). a decrease in age-adjusted CHD mortality in the United States (US) (Xu et al., 2010), and decrease in self-reported CHD (Centers for Disease Control and Prevention, 2011) from 2006 to 2010, CHD remains one of the leading causes of death in the US. (Murphy et al., 2012) CHD risk factors include diabetes (Grossman \& Messerli, 1996), hypercholesterolemia (Wijeysundera et al., 2010), hypertension (Strauer, 1979), and smoking (Scheidt, 1997). Accordingly, risk scores have been developed to enable clinicians to quantify these risk factors from their

Abbreviations: CRF, cardiorespiratory fitness; e-CRF, estimated cardiorespiratory fitness; CHD, coronary heart disease.

* Corresponding author at: 101 Woodruff Circle, Suite 5001, Emory University, Atlanta, GA 30306, United States.

E-mail address: jennifer.gander@emory.edu (J.C. Gander). patients' medical histories in order to provide an estimate of CHD risk (Assmann \& Schulte, 1988; Wilson et al., 1998).

The Framingham Risk Score (FRS) was reported by physicians to be the most widely used CHD risk score (Sposito et al., 2009; Kannel et al., 1976) The FRS was developed from the Framingham Heart Study (Kannel et al., 1976), and a 1998 version by Wilson et al. (1998) categorized the aforementioned risk factors to determine 10-year CHD risk and provide a score sheet for clinical implementation. The FRS' predictive power has persisted through validation in various populations (Kagan et al., 1975; Stampfer et al., 1991) as well as modifications such as the inclusion of apolipoproteins (Ingelsson et al., 2007), C-reactive protein (Pischon et al., 2007), and involuntary job loss (Gallo et al., 2006).

Few studies have examined the association between FRS and cardiorespiratory fitness (CRF) (Gander et al., 2015). The protective effect of CRF on CHD (Chong et al., 1999; Ekelund et al., 1988), and other adverse events has been well documented (Blair et al., 1989a; Sui et al., 2007; Sui et al., 2008; Sieverdes et al., 2010; Gander et al., 2011). Our recent study examining the association between CRF and 10-year CHD risk showed that men with high CRF had a $26 \%$ (HR $=0.75$; $95 \%$ CI $0.56-$ 
0.98) lower risk of CHD compared to men with low CRF, while controlling for an individual's FRS-predicted risk (Gander et al., 2015). A clinical limitation to CRF, however, is the methodologic rigor and associated high costs required to determine an individual's CRF, traditionally determined via a maximal exercise test. For these reasons, researchers have developed methods for estimating a patient's CRF (Nes et al., 2014; Jackson et al., 2012; Coleman et al., 2012). Recently, a 7-item, non-exercise, scale estimating CRF (e-CRF) (Jackson et al., 2012) was developed that incorporates sex, age, body mass index (BMI), waist circumference (WC), resting heart rate (RHR), smoking status, and physical activity (PA). No study has investigated the association between e-CRF and CHD independently or in addition to a CHD risk score, such as the FRS. This study was designed to expand on previous literature by determining the relationship between e-CRF and CHD. A second aim was to evaluate the potential for the e-CRF to add clinical value to the FRS by testing for improvement in predicting 10-year CHD risk.

\section{Methods}

\subsection{Study population}

This study focused on men from the Aerobics Center Longitudinal Study (ACLS) prospective cohort. The ACLS participants were recruited from patients attending the Cooper Clinic in Dallas, TX for a preventive medical examination and health behavior counseling. The participants completed a baseline examination at the Cooper Clinic from January 1 , 1979 through December 31, 2002. Women were excluded from analyses due to a low number of CHD events $(n=45)$. The five inclusion criteria for men were 1) age at baseline was between 30 and 74 years, 2) $\mathrm{BMI} \geq 18.5 \mathrm{~kg} / \mathrm{m}^{2}, 3$ ) free of a previous $\mathrm{CHD}$, cancer, or stroke diagnosis at baseline, 4) reached an age-predicted maximal exercise heart rate $\geq 85 \%$ at each visit, and 5 ) had complete data with a minimum of 1 year of follow-up. The Cooper Clinic Institutional Review Board reviewed and approved the ACLS protocol annually. Fig. 1 displays the inclusion and exclusion criteria for this study.

\subsection{Clinical examination}

Standardized protocols were followed by trained technicians at every clinical exam. Personal and family medical histories were taken during the baseline examination. Other clinical baseline measures included a 12-hour fasting cholesterol and glucose measurement, blood pressure assessment, electrocardiogram, anthropometric measurements, and a maximal exercise test (Blair et al., 1989b; Blair et al., 1995; Blair et al., 1996). A standardized questionnaire was used to capture an individual's current smoking status and medical history.

\subsection{Measures}

\subsubsection{Definition of outcome}

CHD was defined either by self-reported myocardial infarction, bypass surgery, coronary balloon, angioplasty, or stent placement, or by CHD mortality. Self-reported history of CHD was collected through a mail-back survey administered in 1982, 1986, 1990, 1995, 1999, and 2004 and this method of event identification has been described before (Sui et al., 2007). The aggregate survey response rate across all survey periods in the ACLS is approximately $65 \%$. Nonresponse bias is a concern in epidemiologic surveillance, and this issue has been investigated previously (Macera et al., 1990). CHD-specific mortality was determined through linking the ACLS cohort with the National Center for Health Statistic's National Death Index. The primary cause of death was determined by International Classification of Disease Ninth (ICD-9) and Tenth (ICD-10) revisions. CHD mortality was classified with ICD-9 codes 410.0-414.0 and ICD-10 codes I20-I25. In accordance with FRS's follow-up definition, the cut-off for maximum follow-up time for CHD event was 12 years.

\subsubsection{Primary exposure}

Estimated CRF (e-CRF) was expressed in metabolic equivalent of task (MET) units, which were estimated using a 7-item algorithm (Artero et al., 2014). The sex-specific scale is composed of a participant's age, BMI, WC, RHR, two-level PA, and smoking status (smoke). Details on PA were captured through a medical history questionnaire in which participants reported their regular PA for the past 3 months (Kampert et al., 1996; Cheng et al., 2000), and dichotomized in to two levels: none or low, vs. moderate or high physical activity. The accuracy of the developed algorithm was determine by computing the random intercept's square root of the sum and the residual variances (Jackson et al., 2012; O'Connor et al., 2010). These

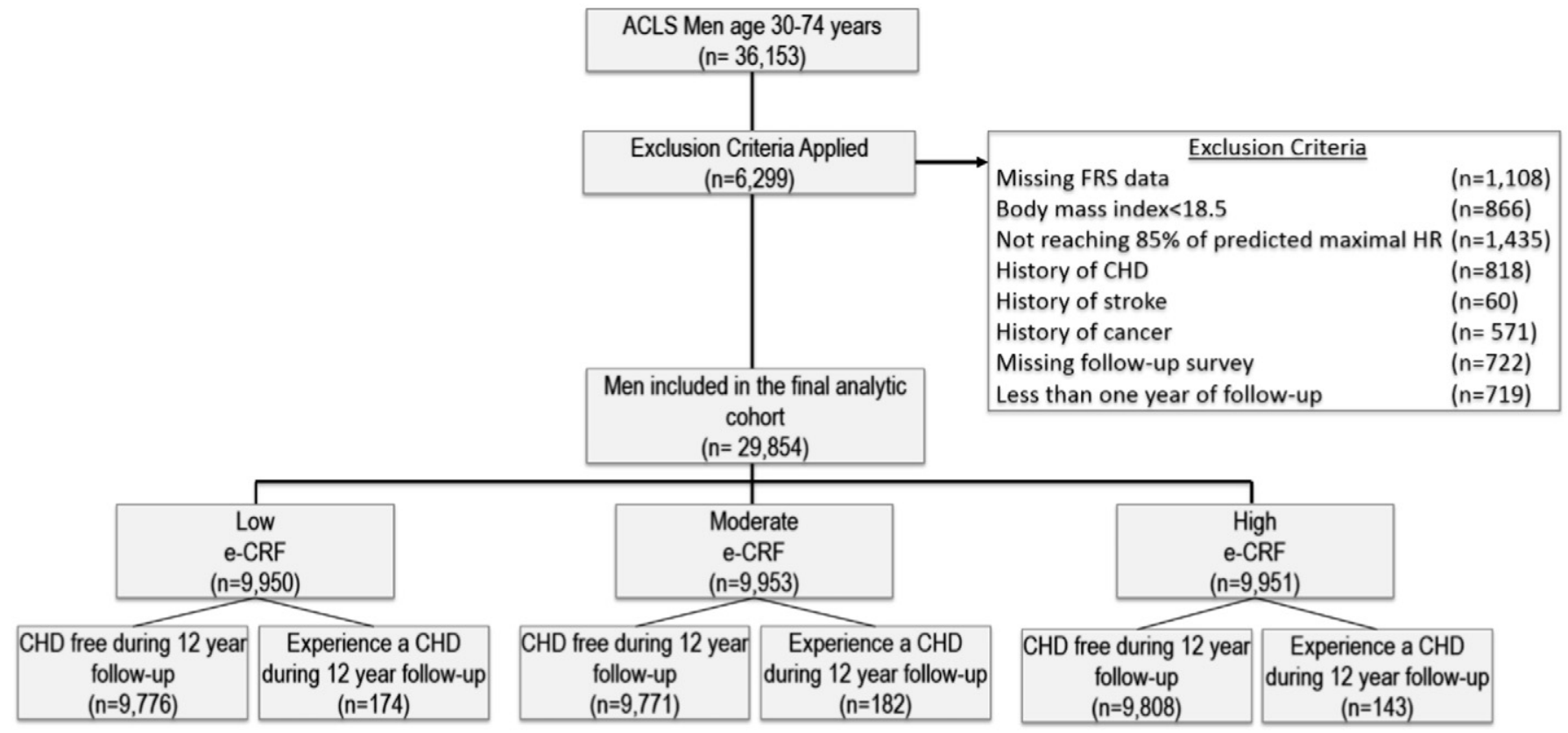

Fig. 1. Aerobics Center Longitudinal Study participants flow diagram. FRS, Framingham Risk score; CHD, coronary heart disease; HR, heart rate. 
variables are applied to Jackson et al.'s algorithm (Jackson et al., 2012) below:

$$
\begin{aligned}
e-C R F= & 21.2870 \\
& +(\text { Age } \times 0.1654)-\left(\text { Age }^{2} \times 0.0023\right) \\
& -(B M I \times 0.2318)-(W C \times 0.0337) \\
& -(R H R \times 0.0390)+(P A \times 0.6351)-(\text { Smoke } \times 0.4263)
\end{aligned}
$$

The estimated METs were categorized into three age-adjusted e-CRF tertiles. This classification is similar to a previous e-CRF study (Jackson et al., 2012).

\subsubsection{Application of Framingham Risk Score (FRS)}

A composite 10-year CHD risk score was generated for each participant using the FRS. The FRS was derived from the Framingham Heart Study and the 1998 modeling (Wilson et al., 1998) to predict 10-year CHD risk. The FRS is a sex-specific and age-adjusted risk score that incorporates categorical variables for blood pressure, total cholesterol, high density lipoprotein cholesterol (HDL-C), diabetes diagnosis, and smoking status. Blood pressure, total cholesterol, and HDL-C were categorized based on FRS definitions (Wilson et al., 1998). Point values were tabulated for each participant based on Wilson et al.'s study (Wilson et al., 1998). Men were stratified based on their level of 10-year CHD risk and men with a 10 -year CHD risk $<10 \%$ (point summation $\leq 5$ points) were classified as 'low' risk and a point summation $>5$ points was categorized as 'moderate or high' risk for CHD (Wilson et al., 1998).

\subsection{Statistical analysis}

Descriptive statistics were calculated for the total study population and stratified by e-CRF. Chi-square tests and Cochran Armitage trend tests were conducted to determine significant differences between the e-CRF levels and each risk factor. Death rate per 10,000 person-years of follow-up was calculated for e-CRF and 10-year FRS predicted CHD Risk. We conducted a sensitivity analysis to determine the correlation between estimated METS and FRS point summation. Crude Cox Proportional Hazard Models, adjusting for baseline examination year, were fit to determine the association between e-CRF and CHD, and the relationship between 10-year FRS predicted CHD risk and actual CHD events. A fully adjusted Cox Proportional Hazard Model was used to determine the relationship between e-CRF, 10-year FRS predicted CHD risk and CHD. The effect of e-CRF on CHD also was investigated on population subsets defined by age, smoking status, hypertension status, and diabetes diagnosis. To investigate if there was a significant interaction between e-CRF and 10-year FRS predicted CHD risk, the male ACLS participants were stratified by 'low' or 'moderate or high' FHS predicted CHD risk and hazard ratios between e-CRF and CHD were calculated. The receiver operating characteristic (ROC) curves were plotted to determine if there was a significant improvement in the predictive accuracy of CHD by augmenting the FRS point summation with e-CRF. The chisquare test determined if there was a significant difference between the models. The Hosmer-Lemeshow statistics was used to assess calibration and goodness of fit; the statistics compares the predictive and observed events but is limited to sample sizes smaller than 10,000 observations (Paul et al., 2013). To control for this limitation, a random sample population was generated from the larger study population to perform this goodness of fit test. Sensitivity analyses determined the correlation between e-CRF and objectively measured CRF, and compared the predictive accuracy of CHD using ROC curves containing FRS point summation and either e-CRF or CRF.(Gander et al., 2015) SAS ${ }^{\circledR}$ version 9.3 was used to perform all descriptive, survival, and predictive modeling analyses.

\section{Results}

There were 499 CHD events among 29,854 men (contributing

\begin{tabular}{|c|c|c|c|c|c|}
\hline Risk factor & $\begin{array}{l}\text { Total Population } \\
(n=29,854) \\
\%\end{array}$ & $\begin{array}{l}\text { Low } \\
\text { e-CRF } \\
(n=9950) \\
\%\end{array}$ & $\begin{array}{l}\text { Middle } \\
\text { e-CRF } \\
(n=9953) \\
\%\end{array}$ & $\begin{array}{l}\text { High } \\
\text { e-CRF } \\
(n=9951) \\
\%\end{array}$ & $\begin{array}{l}\text { Cochran-Armitage Trend } \\
p \text {-value }\end{array}$ \\
\hline Number of CHD events (\%) & $499(1.7)$ & $174(1.8)$ & $182(1.8)$ & $1431.4)$ & 0.08 \\
\hline Mean (SD) age, y & $44.7(8.6)$ & $49.7(8.8)$ & $46.8(8.6)$ & $42.1(8.4)$ & $<0.0001$ \\
\hline Age, range (years) & $30-74$ & $30-74$ & $30-74$ & $30-70$ & \\
\hline Moderate or high 10 -year CHD risk & 2.1 & 3.7 & 1.7 & 0.8 & $<0.0001$ \\
\hline $\mathrm{SBP}$, mean $(\mathrm{SD})^{\mathrm{a}}$ & $120.7(12.9)$ & $124.5(13.1)$ & $120.1(12.2)$ & $117.5(12.5)$ & $<0.0001$ \\
\hline $\mathrm{DBP}$, mean $(\mathrm{SD})^{\mathrm{a}}$ & $81.2(9.4)$ & $84.7(9.5)$ & $80.9(8.9)$ & $78.1(8.7)$ & $<0.0001$ \\
\hline \multicolumn{6}{|l|}{ Blood pressure, $\mathrm{mm} \mathrm{HG}$} \\
\hline Optimal $(\mathrm{SBP}<120, \mathrm{DBP}<80)$ & 28.8 & 16.6 & 29.4 & 40.4 & $<0.0001$ \\
\hline Normal $(120 \leq \mathrm{SBP}<130,80 \leq \mathrm{DBP}<85)$ & 31.8 & 29.2 & 33.7 & 32.6 & $<0.0001$ \\
\hline High normal $(130 \leq \mathrm{SBP}<140,85 \leq \mathrm{DBP}<90)$ & 16.0 & 18.6 & 16.1 & 13.2 & $<0.0001$ \\
\hline Stage I HTN $(140 \leq \mathrm{SBP}<160,90 \leq \mathrm{DBP}<100)$ & 18.7 & 26.9 & 17.4 & 11.9 & $<0.0001$ \\
\hline Stages II-IV HTN & 4.7 & 8.7 & 3.4 & 1.9 & $<0.0001$ \\
\hline \multicolumn{6}{|l|}{$(\mathrm{SBP} \geq 160, \mathrm{DBP} \geq 100)$} \\
\hline Total cholesterol, mg/dL, mean (SD) ${ }^{\mathrm{a}}$ & 208.63 (39.739) & $216.197(41.0532)$ & $216.197(41.0532)$ & $199.578(36.5252)$ & $<0.0001$ \\
\hline$<160$ & 9.2 & 6.8 & 8.2 & 12.5 & $<0.0001$ \\
\hline $160-199$ & 34.1 & 28.7 & 33.3 & 40.3 & $<0.0001$ \\
\hline $200-239$ & 36.9 & 38.5 & 37.9 & 34.3 & $<0.0001$ \\
\hline $240-279$ & 15.3 & 19.6 & 15.7 & 10.6 & $<0.0001$ \\
\hline$\geq 280$ & 4.5 & 6.4 & 4.8 & 2.3 & $<0.0001$ \\
\hline HDL-C, mg/dL, mean (SD) ${ }^{a}$ & $46.1(12.1)$ & $42.0(10.7)$ & $45.9(11.5)$ & $50.4(12.6)$ & $<0.0001$ \\
\hline$<35$ & 15.6 & 24.8 & 14.2 & 7.9 & $<0.0001$ \\
\hline $35-44$ & 34.1 & 39.9 & 36.0 & 26.5 & $<0.0001$ \\
\hline $45-49$ & 15.6 & 13.8 & 16.7 & 16.2 & $<0.0001$ \\
\hline $50-59$ & 21.4 & 15.1 & 21.4 & 27.7 & $<0.0001$ \\
\hline$\geq 60$ & 13.3 & 6.5 & 11.7 & 21.7 & $<0.0001$ \\
\hline Diabetes & 1.4 & 2.8 & 0.9 & 0.6 & $<0.0001$ \\
\hline Current smoker & 16.6 & 24.4 & 17.1 & 8.1 & $<0.0001$ \\
\hline
\end{tabular}
248,890 person-years of observation) (Fig. 1). Table 1 displays the

Table 1

Demographics of participants stratified by estimated cardiorespiratory fitness (e-CRF).

Abbreviations: DBP, diastolic blood pressure; e-CRF, estimated cardiorespiratory fitness; HDL-C, high density lipoprotein- cholesterol; SBP, systolic blood pressure.

a Categorization for blood pressure, total cholesterol, and HDL-C were based on the 1998 Framingham Risk Score classification (Wilson et al., 1998). 
Table 2

Adjusted survival risks for coronary heart disease (CHD) events by estimated cardiorespiratory fitness (e-CRF) and 10-year CHD risk group.

\begin{tabular}{|c|c|c|c|c|c|}
\hline & $\mathrm{N}$ & $\begin{array}{l}\text { Number of } \\
\text { deaths }\end{array}$ & $\begin{array}{l}\text { Death } \\
\text { rate }^{\mathrm{a}}\end{array}$ & $\begin{array}{l}\text { HR } \\
(95 \% \mathrm{CI})^{\mathrm{b}}\end{array}$ & $\begin{array}{l}\text { HR } \\
(95 \% \mathrm{CI})^{\mathrm{c}}\end{array}$ \\
\hline \multicolumn{6}{|l|}{$\begin{array}{l}\text { Estimated CRF } \\
\text { (e-CRF) }\end{array}$} \\
\hline Low & 9950 & 174 & 31.93 & 1.00 (ref) & 1.00 (ref) \\
\hline Moderate & 9953 & 182 & 18.90 & $\begin{array}{l}0.93 \\
(0.76,1.14)\end{array}$ & $\begin{array}{l}0.99 \\
(0.81,1.22)\end{array}$ \\
\hline High & 9951 & 143 & 10.31 & $\begin{array}{l}0.64 \\
(0.51,0.80)\end{array}$ & $\begin{array}{l}0.71 \\
(0.56,0.88)\end{array}$ \\
\hline $\begin{array}{l}P \text { value for trend } \\
10 \text {-year CHD risk }\end{array}$ & & & & $<0.001$ & 0.003 \\
\hline Low & 29,241 & 447 & 18.34 & 1.00 (ref) & 1.00 (ref) \\
\hline Moderate or high & 613 & 52 & 102.58 & $\begin{array}{l}5.59 \\
(4.20,7.45)\end{array}$ & $\begin{array}{l}5.25 \\
(3.92,7.01)\end{array}$ \\
\hline
\end{tabular}

Abbreviations: CHD, coronary heart disease; CI, confidence interval; HR, hazard ratio.

a Deaths per 10,000 person-years of follow-up adjusted for examination year.

b Adjusted for examination year.

c Further adjusted e-CRF for 10-year CHD risk or 10-year CHD risk for e-CRF.

comparisons between men stratified by their e-CRF. Men with low eCRF had a higher proportion of CHD events compared to high-fit men. Men with low e-CRF also were less likely to have optimal or normal blood pressure compared to men with moderate or high e-CRF. Highfit men were more likely to have increased levels of HDL-C $\geq 60 \mathrm{mg} / \mathrm{dL}$, be nondiabetic, and be a nonsmoker compared to low- or moderate fit men.
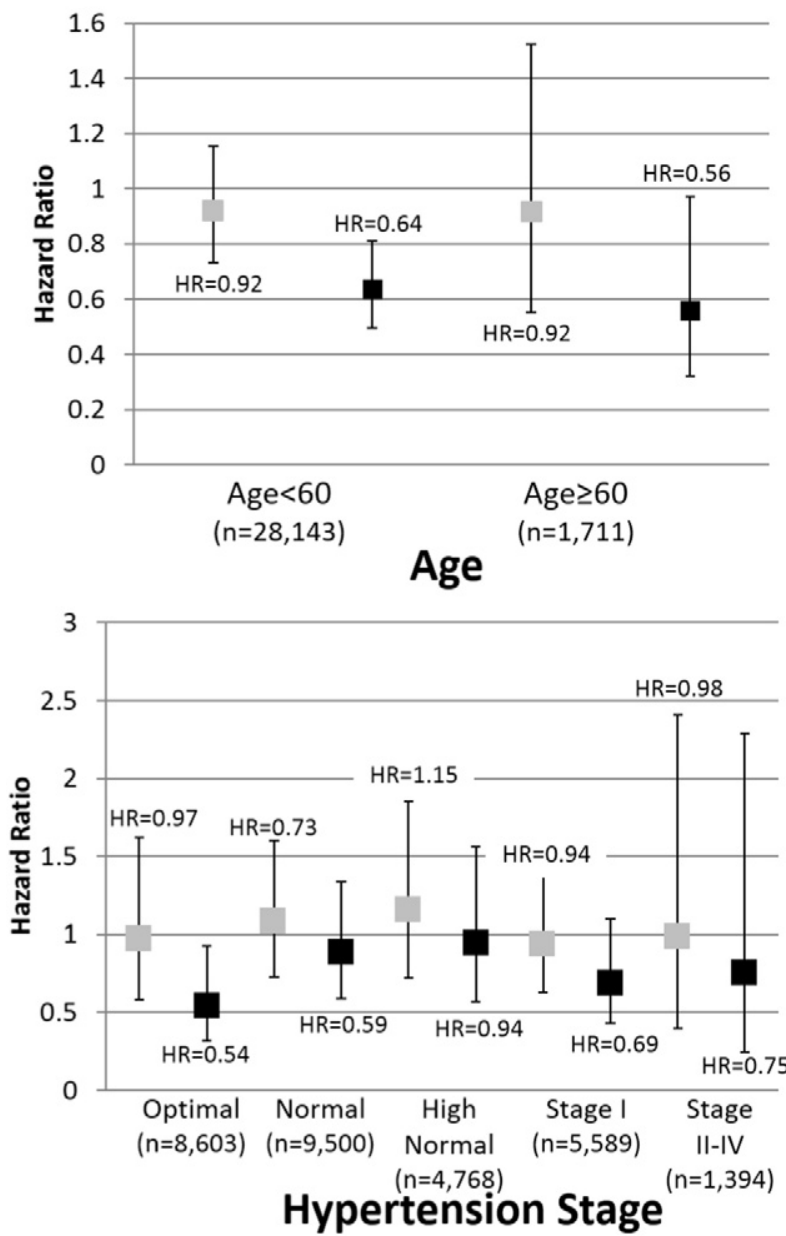

Our sensitivity analysis testing the correlation between estimated METS and FRS point summation reported a significant inverse correlation (Spearman $r=-0.54, p<0.001$ ). Crude survival analysis, adjusted for baseline examination year, reported that both e-CRF and 'moderate or high' 10-year FRS predicted CHD risk were statistically significant with CHD (Table 2). In the crude survival analysis, men with high eCRF had a 36\% (HR $=0.64 ; 95 \% \mathrm{CI} 0.51,0.80)$ lower CHD risk compared to low-fit men ( $p$-value for trend $<0.001$ ). The significant association between e-CRF and CHD remained in a subsequent model controlling for 'moderate or high' 10-year FRS predicted CHD risk, although effect size was slightly attenuated. The significant protective effect between e-CRF and CHD was also found in subpopulations of male ACLS cohort members. Fig. 2 shows that among men age $\geq 60$ years, high-fit men had a $44 \%$ ( $\mathrm{HR}=0.56 ; 95 \% \mathrm{Cl} 0.32,0.97)$ lower risk for CHD compared to low-fit men. A similar association between high-fit men and CHD risk was found among nonsmokers ( $\mathrm{HR}=0.62 ; 95 \% \mathrm{CI} 0.48,0.79$ ).

Fig. 3 reports the interaction between e-CRF and FRS predicted CHD. In analyses stratified by 'low' and 'moderate or high' FRS predicted CHD risk, men with 'low' 10-year FRS predicted CHD risk and high e-CRF have a $28 \%(\mathrm{HR}=0.72 ; 95 \% \mathrm{CI} 0.57,0.91)$ lower risk of CHD compared to men with low 10-year FRS predicted CHD risk and low e-CRF. Among men with 'moderate or high' 10-year FRS predicted CHD risk, men with moderate e-CRF were $38 \%$ ( $\mathrm{HR}=0.62 ; 95 \% \mathrm{CI} 0.32,1.22$ ) lower risk for CHD compared to men with low e-CRF. High e-CRF was associated with a protective effect $(\mathrm{HR}=0.69 ; 95 \% \mathrm{CI} 0.31,1.51)$ of $\mathrm{CHD}$ in men with 'moderate or high' FRS predicted CHD risk, although neither relationship was statistically significant.
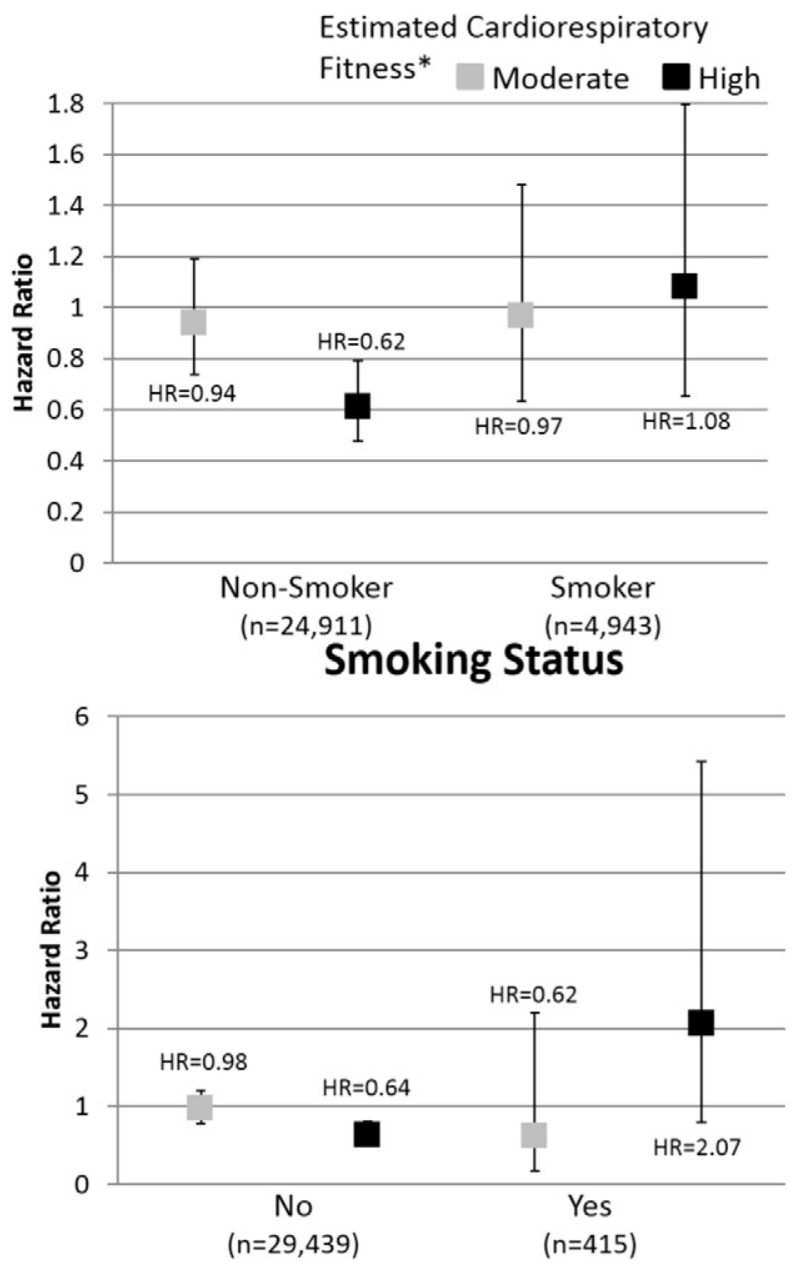

Diabetes Diagnosis

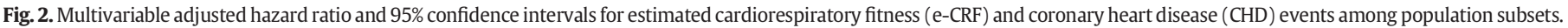




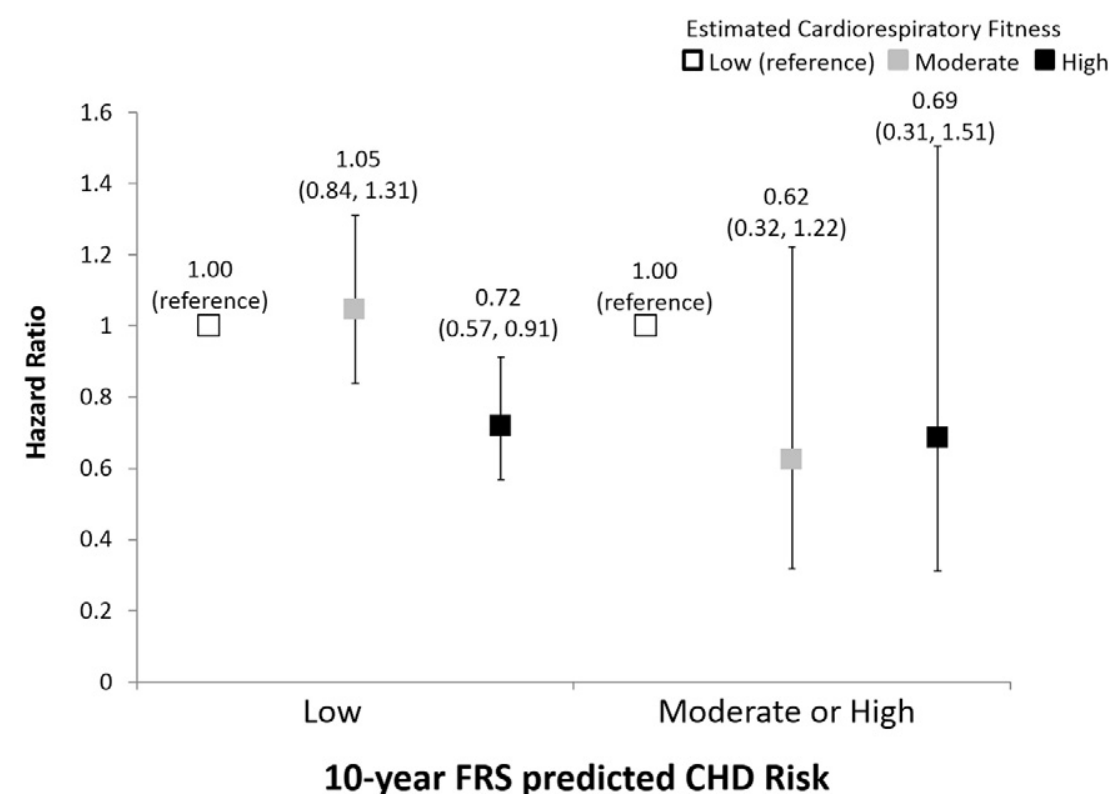

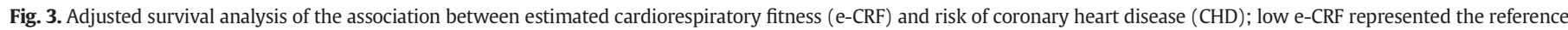
group.

The ROC curves were plotted for 'FRS point summation only' model and the 'FRS point summation with e-CRF' (Fig. 4). The Area Under the Curve was higher for the 'FRS point summation with e-CRF' (c-statistic $=0.7987 ; 95 \% \mathrm{CI} 0.7813,0.8161$ ) compared to the model 'FRS point summation only' (c-statisti $=0.7972 ; 95 \%$ CI 0.7798, 0.8146). However, there was no significant difference in predictive power between the models ( $p$-value $=0.90$ ) and the goodness of fit test reported that the predicted events were not different from the observed events with a Hosmer-Lemeshow $p$-value $>0.05$. The sensitivity analysis to determine the correlation between e-CRF and objectively measured CRF reported a significant correlation between e-CRF $(R=0.54 ; p<0.001)$ and similar predictive accuracy (CRF's c-statistic $=0.7976)$, and no significant difference in predictive power $(p=0.93)$.

\section{Discussion}

Men with high e-CRF were at a significantly lower risk for CHD compared to men with low e-CRF (reference group). Among men with 'low'

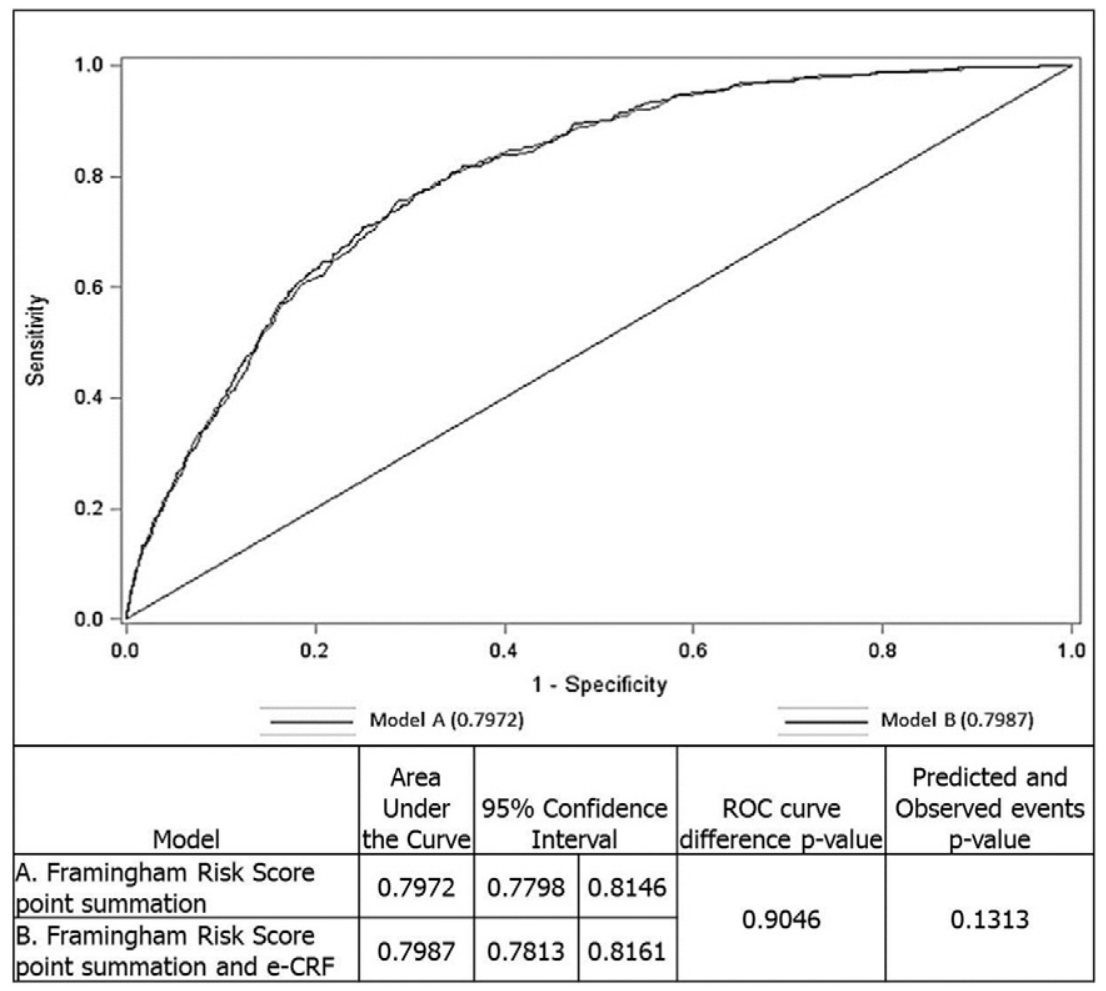

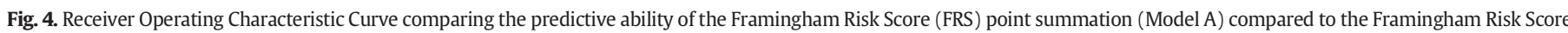
point summation and estimated cardiorespiratory fitness (e-CRF) (Model B). 
FRS predicted risk for CHD, high-fit men had a significantly lower risk for CHD compared to men with e-CRF. To our knowledge, this is the first study to examine the protective effect of e-CRF on CHD among men with 'moderate or high' risk for CHD by the FRS assessment.

Cardiorespiratory fitness is a protective factor for all-cause mortality,(Blair et al., 1989a; Wei et al., 2000) CHD mortality,(Chong et al., 1999; Ekelund et al., 1988; Wilson et al., 1998) CHD incidence,(Oja et al., 1974) and diabetes incidence,(Sui et al., 2008) but is not routinely collected during clinical visits. Estimated CRF provides a clinically feasible alternative, can be captured by using a simple algorithm,(Nes et al., 2014; Artero et al., 2014; O'Connor et al., 2010) and has been shown to be a protective factor of all-cause mortality in a cohort of healthy men and women,(Nauman et al., 2016) all-cause mortality in adults with elevated inflammation index,(Edwards \& Loprinzi, 2017) and cardiovascular-specific mortality.(Nauman et al., 2016) Our study reports a significant protective association between e-CRF and CHD, congruent with previous reports on e-CRF's protective effect on other cardiovascular outcomes.(Nes et al., 2014; Artero et al., 2014; Stamatakis et al., 2012) Nauman et al. reported that e-CRF was strongly associated with an inverse association of cardiovascular disease mortality in a healthy Norwegian sample of 38,480 men and women.(Nauman et al., 2016) The study reported that the addition of e-CRF resulted in net gain reclassification of cardiovascular-specific mortality of $2.8 \%$ and $6.8 \%$ in men and women, respectively, independent of traditional cardiovascular disease risk factors. Our study reports similar protective effects, in men, of e-CRF on CHD mortality in the overall cohort and stratified by various patient characteristics. Furthermore, e-CRF's protective association of CHD mortality is independent of 10-year CHD risk measured by the FRS.

The FRS has been validated in various populations with similar results to ours (Gander et al., 2014). Male physicians in the US, enrolled in the Physician's Health Study, reported their CHD risk factors through a questionnaire at enrollment and completed follow-up surveys every 6 months to capture CHD incidence (Stampfer et al., 1991). The study found similar risk factors associated with CHD as those reported in the Framingham Heart Study, with the exception of smoking status. Additionally, D'Agostino et al. conducted a comparison analysis to determine the level of agreement between the FRS applied to the Framingham Heart Study cohort and the FRS applied to non-Framingham Heart Study populations. They concluded that the level of agreement was reasonably sound between the predicted and actual CHD events, with the exception of the study implemented using the Japanese-American cohort (D'Agostino et al., 2001). More specifically, when the FRS was applied to the ACLS cohort the ROC reported FRS had a strong predictive power of CHD mortality ( $c=0.7697$; 95\% CI 0.7523, 0.7871) (Gander et al., 2014). While the e-CRF algorithm overlaps with the FRS by including age, sex, and smoking status, Artero et al.'s calculation additionally includes BMI, waist circumference, resting heart rate, and physical activity,(Artero et al., 2014). each variable known to be independently associated with CHD and CHD-mortality.(Fitzgerald \& Jarrett, 1992; Jousilahti et al., 1996; de Hollander et al., 2013; Dyer et al., 1980; Gillman et al., 1993; Rexrode et al., 1998; Rimm et al., 1995; Bijnen et al., 1994; Batty \& Lee, 2004; Berlin \& Colditz, 1990; Slattery et al., 1989). The addition of the aforementioned variables may explain eCRF's incremental, additional protective effect on CHD mortality and slight improvement on FRS' predictive power. In the current report, when we stratified our cohort by FRS 10-year CHD risk ('low' vs 'moderate or high'), among those with 'low' CHD risk, men with high e-CRF appeared to have a lower risk of CHD mortality compared to men with low e-CRF.

A study measuring the association of CRF on CHD risk reported CRF'S significant protective effect on CHD while controlling for individuals' FRS predicted risk (Gander et al., 2015). That study was conducted in men from the ACLS cohort and reported that in the model adjusting for FRS predicted risk, high-fit men had 26\% (HR $=0.74 ; 95 \% \mathrm{CI} 0.56$, 0.98) lower risk for CHD compared to low-fit men. A clinical limitation to that study however, is the rigorous methodology required to obtain an individual's CRF. The American Heart Association reported on the importance of collecting CRF and the additional information measured CRF could provide for patient management.(Ross et al., 2016). Our study provides a clinically feasible method to obtain e-CRF which offers the predictive capability of traditionally measured CRF (Jurca et al., 2005). while reducing the cost/burden to the patient and clinician.(Myers, 2014). As stated above, improvements in CRF as calculated by e-CRF may lead to additional improvements in other CHD risk factors, such as hypertension and glycemic control and could potentially be considered as part of primary and secondary prevention (Ross et al., 2016; Vuori et al., 2013; Swift et al., 2013; Lavie et al., 2015).

\subsection{Study limitations}

The limitations of this study should be noted and considered when determining generalizability. Due to the small number of CHD events occurring in women, only men were included in the present analysis. Future research should investigate the association between e-CRF and $\mathrm{CHD}$ in asymptomatic women. The non-significant association between e-CRF and CHD among stage II-IV hypertensive men may be due to the small proportions; therefore, generalizations toward this group should also be made cautiously. It also should be noted that the ACLS cohort consists predominately of non-Hispanic White individuals from middle to upper socioeconomic status who were relatively young (i.e., with a mean age of 42 years). Although this limitation may be considered a strength because of its tendency to improve internal validity while exerting inherent control for possible demographic confounders, generalizations and implementations of e-CRF should be made cautiously. In addition, the ACLS cohort is a relatively healthy population with respect to CHD and 10-year CHD risk. While previous publications have shown the predictive power of FRS within the ACLS cohort,(Gander et al., 2015; Gander et al., 2014) generalizations should be made with caution. Specifically, our stratified, adjusted associations between 'moderate or high' 10-year FRS predicted CHD risk and e-CRF showed a protective effect, though not statistically significant, possibly due to a limited number of events within these sub-populations. Future studies should investigate this association among a population with more CHD events among 'moderate or high' 10-year FRS predicted risk participants. Our sensitivity analyses reported a statistically significant inverse correlation between non-exercise estimated METS and FRS point summation and may be considered a limitation. However, these variables were both significant predictors of mortality in the fully adjusted regression model and they were both included in the model. Physical activity was collected through a medical history questionnaire that requests the participant to report their physical activity habits during the past 3 months. (Jackson et al., 2012; Cheng et al., 2000) While the self-reported physical activity may be limited by recall bias, literature shown successful utilization of physical activity questionnaires within clinical practice that led to a higher number of patients discussing exercise with their physician (Grant et al., 2014).

\section{Conclusion}

Our study found that among men with 'low' 10-year FRS predicted CHD risk, those with high e-CRF had a lower risk for CHD compared to men with low fitness. Increasing awareness through early quantification of a patient's risk for CHD is important for CHD prevention. The FRS is a validated tool that enables physicians to assess an individual's risk, and our results suggest that assessing e-CRF may add considerably to the clinicians' overall risk assessment for CHD. The results of this 7item survey (age, sex, WC, BMI, PA, RHR, and smoking status) can be quickly and easily collected during a clinical exam by paramedical staff, may help clinicians predict adverse CHD events, and provide clinicians ammunition for the promotion of PA and exercise training for improving CRF and CHD risk. 


\section{Role of funding source}

Dr. Gander was supported by a National Research Service Award from the National Institute of Diabetes and Digestive and Kidney Diseases (F32DK10719). Dr. Hébert was supported by an Established Investigator Award in Cancer Prevention and Control from the Cancer Training Branch of the National Cancer Institute (K05 CA136975).

Research for this study was supported by National Institutes of Health grants AG06945, HL62508, and DK088195.

The content is solely the responsibility of the authors and does not necessarily represent the official views of the National Institutes of Health.

We thank the Cooper Clinic physicians and technicians for collecting the baseline data, and staff at the Cooper Institute for data entry and data management. JGander would like to thank her Emory mentor, Rachel Patzer, for her support.

\section{Disclosures}

None.

\section{Conflict of interest}

There is no conflict of interests to disclose that may influence the research presented in the submitted manuscript. This material has not been previously published and is not under consideration elsewhere. All authors have participated, read, and approved the research and development of the manuscript. All financial support has been stated in the Acknowledgements section.

\section{Acknowledgments}

\section{Author affiliation:}

Division of Transplantation, Department of Surgery, Emory School of Medicine, Emory University, Atlanta, Georgia (Jennifer Gander);

Department of Epidemiology and Biostatistics, Arnold School of Public Health, University of South Carolina, Columbia, South Carolina (Linda Hazlett, James Hébert, Steven Blair); Department of Exercise Science, Arnold School of Public Health, University of South Carolina, Columbia, South Carolina (Xuemei Sui, Steven Blair); South Carolina Statewide Cancer Prevention and Control Program, University of South Carolina, Columbia, South Carolina (James Hébert).

\section{References}

Artero, E.G., Jackson, A.S., Sui, X., et al., 2014. Longitudinal algorithms to estimate cardiorespiratory fitness: associations with nonfatal cardiovascular disease and diseasespecific mortality. J. Am. Coll. Cardiol. 63 (21), 2289-2296.

Assmann, G., Schulte, H., 1988. The prospective cardiovascular Münster (PROCAM) study: prevalence of hyperlipidemia in persons with hypertension and/or diabetes mellitus and the relationship to coronary heart disease. Am. Heart J. 116 (6), 1713-1724.

Batty, G.D., Lee, I.-M., 2004. Physical activity and coronary heart disease. British Medical Journal Publishing Group.

Bennett, K., Kabir, Z., Unal, B., et al., 2006. Explaining the recent decrease in coronary heart disease mortality rates in Ireland, 1985-2000. J. Epidemiol. Community Health 60 (4), 322-327.

Berlin, J.A., Colditz, G.A., 1990. A meta-analysis of physical activity in the prevention of coronary heart disease. Am. J. Epidemiol. 132 (4), 612-628.

Bijnen, F., Caspersen, C., Mosterd, W., 1994. Physical inactivity as a risk factor for coronary heart disease: a WHO and International Society and Federation of Cardiology position statement. Bull. World Health Organ. 72 (1), 1.

Blair, S.N., Cooper, K.H., Gibbons, L.W., 1989a. Physical fitness and all-cause mortality of healthy men and women. JAMA 262 (0), 2395-2401.

Blair, S.N., Kannel, W.B., Kohl, H.W., Goodyear, N., Wilson, P.W., 1989b. Surrogate measures of physical activity and physical fitness evidence for sedentary traits of resting tachycardia, obesity, and low vital capacity. Am. J. Epidemiol. 129 (6), 1145-1156.

Blair, S.N., Kohl, H., Barlow, C.E., Paffenbarger, R., Gibbons, L.W., Macera, C.A., 1995. Changes in physical fitness and all-cause mortality. JAMA 273 (14), 1093-1098.

Blair, S.N., Kampert, J.B., Kohl III, H.W., et al., 1996. Influences of cardiorespiratory fitness and other precursors on cardiovascular disease and all-cause mortality in men and women. JAMA 276 (3), 205-210.
Centers for Disease Control and Prevention, 2011. Prevalence of coronary heart diseaseUnited States, 2006-2010. MMWR Morbidity and mortality weekly report. 60(40), p. 1377.

Cheng, Y., Macera, C.A., Davis, D.R., Ainsworth, B.E., Troped, P.J., Blair, S.N., 2000. Physical activity and self-reported, physician-diagnosed osteoarthritis: is physical activity a risk factor? J. Clin. Epidemiol. 53 (3), 315-322.

Chong, D.L., Blair, S.N., Jackson, A.S., 1999. Cardiorespiratory fitness, body composition, and all-cause and cardiovascular disease mortality in men. Am. J. Clin. Nutr. 69 (3), 373-380.

Coleman, K.J., Ngor, E., Reynolds, K., et al., 2012. Initial validation of an exercise "vital sign" in electronic medical records. Med. Sci. Sports Exerc. 44 (11), 2071-2076.

D'Agostino Sr., R.B., Grundy, S., Sullivan, L.M., Wilson, P., 2001. Validation of the Framingham coronary heart disease prediction scores. JAMA 286 (2), 180-187.

Dyer, A.R., Persky, V., Stamler, J., et al., 1980. Heart rate as a prognostic factor for coronary heart disease and mortality: findings in three Chicago epidemiologic studies. Am. J. Epidemiol. 112 (6), 736-749.

Edwards, M.K., Loprinzi, P.D., 2017. The association of estimated cardiorespiratory fitness on mortality risk among those with an elevated gamma gap. Int. J. Cardiol. 227, 508-510.

Ekelund, L.-G., Haskell, W.L., Johnson, J.L., Whaley, F.S., Criqui, M.H., Sheps, D.S., 1988 Physical fitness as a predictor of cardiovascular mortality in asymptomatic North American men. N. Engl. J. Med. 319 (21), 1379-1384

Fitzgerald, A., Jarrett, R., 1992. Body weight and coronary heart disease mortality: an analysis in relation to age and smoking habit. 15 years follow-up data from the Whitehall Study. Int. J. Obes. Relat. Metab. Disord.: Journal of the International Association for the Study of Obesity 16 (2), 119-123.

Gallo, W.T., Teng, H.-M., Falba, T.A., Kasl, S.V., Krumholz, H.M., Bradley, E.H., 2006. The impact of late career job loss on myocardial infarction and stroke: a 10 year follow up using the health and retirement survey. Occup. Environ. Med. 63 (10), 683-687.

Gander, J., Lee, D.-C., Sui, X., Hébert, J.R., Hooker, S.P., Blair, S.N., 2011. Self-rated health status and cardiorespiratory fitness as predictors of mortality in men. Br. J. Sports Med. 45 (14), 1095-1100.

Gander, J., Sui, X., Hazlett, L.J., Cai, B., Hebert, J.R., Blair, S.N., 2014. Factors related to coronary heart disease risk among men: validation of the framingham risk score. Prev. Chronic Dis. 11, E140.

Gander, J.C., Sui, X., Hébert, J.R., et al., 2015. Association of Cardiorespiratory Fitness with Coronary Heart Disease in Asymptomatic Men. Paper presented at. Mayo Clinic Proceedings.

Gillman, M.W., Kannel, W.B., Belanger, A., D'Agostino, R.B., 1993. Influence of heart rate on mortality among persons with hypertension: the Framingham study. Am. Heart J. 125 (4), 1148-1154.

Grant, R.W., Schmittdiel, J.A., Neugebauer, R.S., Uratsu, C.S., Sternfeld, B., 2014. Exercise as a vital sign: a quasi-experimental analysis of a health system intervention to collect patient-reported exercise levels. J. Gen. Intern. Med. 29 (2), 341-348.

Grossman, E., Messerli, F.H., 1996. Diabetic and hypertensive heart disease. Ann. Intern. Med. 125 (4), 304-310.

de Hollander, E.L., Bogers, R.P., Boshuizen, H.C., et al., 2013. Influence of calendar period on the association between BMI and coronary heart disease: a meta-analysis of 31 cohorts. Obesity 21 (5), 865-880.

Ingelsson, E., Schaefer, E.J., Contois, J.H., et al., 2007. Clinical utility of different lipid measures for prediction of coronary heart disease in men and women. JAMA 298 (7), 776-785.

Jackson, A.S., Sui, X., O'Connor, D.P., et al., 2012. Longitudinal cardiorespiratory fitness algorithms for clinical settings. Am. J. Prev. Med. 43 (5), 512-519.

Jousilahti, P., Tuomilehto, J., Vartiainen, E., Pekkanen, J., Puska, P., 1996. Body weight, cardiovascular risk factors, and coronary mortality. Circulation 93 (7), 1372-1379.

Jurca, R., Jackson, A.S., LaMonte, M.J., et al., 2005. Assessing cardiorespiratory fitness without performing exercise testing. Am. J. Prev. Med. 29 (3), 185-193.

Kagan, A., Gordon, T., Rhoads, G.G., Schiffman, J.C., 1975. Some factors related to coronary heart disease incidence in Honolulu Japanese men: the Honolulu heart study. Int. J. Epidemiol. 4 (4), 271-279.

Kampert, J.B., Blair, S.N., Barlow, C.E., Kohl, H.W., 1996. Physical activity, physical fitness, and all-cause and cancer mortality: a prospective study of men and women. Ann. Epidemiol. 6 (5), 452-457.

Kannel, W.B., McGee, D., Gordon, T., 1976. A general cardiovascular risk profile: the Framingham study. Am. J. Cardiol. 38 (1), 46-51.

Lavie, C.J., Arena, R., Swift, D.L., et al., 2015. Exercise and the cardiovascular system. Circ. Res. 117 (2), 207-219.

Macera, C.A., Jackson, K.L., Davis, D.R., Kronenfeld, J.J., Blair, S.N., 1990. Patterns of non-response to a mail survey. J. Clin. Epidemiol. 43 (12), 1427-1430.

Murphy, S.L., Xu, J., Kochanek, K.D., 2012. National vital statistics reports. Natl Vital Stat Rep. 60 (4), 1.

Myers, J., 2014. New American Heart Association/American College of Cardiology Guidelines on Cardiovascular Risk. Paper presented at. Mayo Clinic Proceedings.

Nauman, J., Nes, B.M., Lavie, C.J., et al., 2016. Prediction of Cardiovascular Mortality by Estimated Cardiorespiratory Fitness Independent of Traditional Risk Factors: The HUNT Study. Paper presented at. Mayo Clinic Proceedings.

Nes, B.M., Vatten, L.J., Nauman, J., Janszky, I., Wisløff, U., 2014. A simple nonexercise model of cardiorespiratory fitness predicts long-term mortality. Med. Sci. Sports Exerc. 46 (6), 1159-1165.

O'Connor, D.P., Bray, M.S., McFarlin, B.K., Sailors, M.H., Ellis, K.J., Jackson, A.S., 2010. Generalized equations for estimating DXA percent fat of diverse young women and men: the TIGER study. Med. Sci. Sports Exerc. 42 (10), 1959.

Oja, P., Teräslinna, P., Partanen, T., Kärävä, R., 1974. Feasibility of an 18 months' physical training program for middle-aged men and its effect on physical fitness. Am. J. Public Health 64 (5), 459-465. 
Paul, P., Pennell, M.L., Lemeshow, S., 2013. Standardizing the power of the HosmerLemeshow goodness of fit test in large data sets. Stat. Med. 32 (1), 67-80.

Pischon, T., Möhlig, M., Hoffmann, K., et al., 2007. Comparison of relative and attributable risk of myocardial infarction and stroke according to C-reactive protein and low-density lipoprotein cholesterol levels. Eur. J. Epidemiol. 22 (7), 429-438.

Rexrode, K.M., Carey, V.J., Hennekens, C.H., et al., 1998. Abdominal adiposity and coronary heart disease in women. JAMA 280 (21), 1843-1848.

Rimm, E.B., Stampfer, M.J., Giovannucci, E., et al., 1995. Body size and fat distribution as predictors of coronary heart disease among middle-aged and older US men. Am. J. Epidemiol. 141 (12), 1117-1127.

Rodriguez, T., Malvezzi, M., Chatenoud, L., et al., 2006. Trends in mortality from coronary heart and cerebrovascular diseases in the Americas: 1970-2000. Heart 92 (4), 453-460.

Ross, R., Blair, S.N., Arena, R., et al., 2016. Importance of assessing cardiorespiratory fitness in clinical practice: a case for fitness as a clinical vital sign: a scientific statement from the American Heart Association. Circulation CIR. 0000000000000461.

Scheidt, S., 1997. Changing mortality from coronary heart disease among smokers and nonsmokers over a 20 -year interval. Prev. Med. 26 (4), 441-446.

Sieverdes, J.C., Sui, X., Lee, D.-C., et al., 2010. Physical activity, cardiorespiratory fitness and the incidence of type 2 diabetes in a prospective study of men. Br. J. Sports Med. 44 (4), 238-244.

Slattery, M.L., Jacobs, D.R., Nichaman, M.Z., 1989. Leisure time physical activity and coronary heart disease death. The US Railroad Study. Circulation 79 (2), 304-311.

Sposito, A.C., Ramires, J.A., Jukema, J.W., et al., 2009. Physicians' attitudes and adherence to use of risk scores for primary prevention of cardiovascular disease: cross-sectional survey in three world regions. Curr. Med. Res. Opin. 25 (5), 1171-1178.

Stamatakis, E., Hamer, M., O'Donovan, G., Batty, G.D., Kivimaki, M., 2012. A non-exercise testing method for estimating cardiorespiratory fitness: associations with all-cause and cardiovascular mortality in a pooled analysis of eight population-based cohorts. Eur. Heart J., ehs097
Stampfer, M.J., Sacks, F.M., Salvini, S., Willett, W.C., Hennekens, C.H., 1991. A prospective study of cholesterol, apolipoproteins, and the risk of myocardial infarction. N. Engl. J. Med. 325 (6), 373-381.

Strauer, B.-E., 1979. Myocardial oxygen consumption in chronic heart disease: role of wall stress, hypertrophy and coronary reserve. Am. J. Cardiol. 44 (4), 730-740.

Sui, X., LaMonte, M.J., Blair, S.N., 2007. Cardiorespiratory fitness as a predictor of nonfatal cardiovascular events in asymptomatic women and men. Am. J. Epidemiol. 165 (12), $1413-1423$.

Sui, X., Hooker, S.P., Lee, I.-M., et al., 2008. A prospective study of cardiorespiratory fitness and risk of type 2 diabetes in women. Diabetes Care 31 (3), 550-555.

Swift, D.L., Lavie, C.J., Johannsen, N.M., et al., 2013. Physical activity, cardiorespiratory fitness, and exercise training in primary and secondary coronary prevention. Circ. J.: Official Journal of the Japanese Circulation Society 77 (2), 281-292.

Vuori, I.M., Lavie, C.J., Blair, S.N., 2013. Physical Activity Promotion in the Health Care System. Paper presented at. Mayo Clinic Proceedings.

Wei, M., Gibbons, L.W., Kampert, J.B., Nichaman, M.Z., Blair, S.N., 2000. Low cardiorespiratory fitness and physical inactivity as predictors of mortality in men with type 2 diabetes. Ann. Intern. Med. 132 (8), 605-611.

Wijeysundera, H.C., Machado, M., Farahati, F., et al., 2010. Association of temporal trends in risk factors and treatment uptake with coronary heart disease mortality, 19942005. JAMA 303 (18), 1841-1847.

Wilson, P.W., D'Agostino, R.B., Levy, D., Belanger, A.M., Silbershatz, H., Kannel, W.B., 1998. Prediction of coronary heart disease using risk factor categories. Circulation 97 (18), 1837-1847.

Xu, J., Kochanek, K.D., Murphy, S.L., Tejada-Vera, B., 2010. Deaths: final data for 2007. Natl Vital Stat Rep. 58 (19), 1-136. 